\title{
Calculations of two-dimensional foveal retinal images in real eyes
}

\author{
Pablo Artal \\ Instituto de Optica, Consejo Superior de Investigaciones Científicas, Serrano 121, 28006 Madrid, Spain
}

\begin{abstract}
Received January 23, 1989; second revised manuscript received October 4, 1989; accepted February 28, 1990
Foveal point-spread functions are computed from experimental wave-aberration data for individual emmetropic subjects. The effects of pupil size and image focus are considered in the calculations. Foveal images of extended test objects are generated from the point-spread functions corresponding to different image-quality situations. Wiener-filtered test objects are also computed to obtain a partial compensation of the spatial degradation introduced by the eye's optical system in the visual process.
\end{abstract}

\section{INTRODUCTION}

The optics of the eye play an important role in the spatial information processing performed by the human visual system. The typical contribution to foveal imagery is well understood, but complete characterization of the imaging process for individual subjects has not been possible, particularly as far as two-dimensional analysis is concerned. In this paper, foveal retinal images computed under different image-quality conditions are presented. These have been calculated by using two-dimensional image-quality results obtained by a method proposed in recent papers. ${ }^{1,2}$

The method is based on a hybrid optical-digital technique $^{1}$ that, by using a modified double-pass setup, obtains the retinal image of a point test [i.e., the point-spread function (PSF) of the eye] and the two-dimensional opticaltransfer function (OTF) in foveal vision. The method was extended by developing a phase-retrieval procedure ${ }^{2}$ to compute the wave-aberration function from the foveal PSF data. The experimental image-quality results, obtained for a limited number of subjects, always show a significant number of asymmetric and irregular aberrations. Therefore the phase of the OTF, the phase-transfer function, is not zero for spatial frequencies that are important for visual processing. Two-dimensional phase-transfer functions were directly computed from the retinal images of a point, and their effects on the PSF and wave aberration were analyzed. ${ }^{3}$ Knowledge of the wave aberration permits objective study of several aspects of the optical performance of the eye. Some of these have already been explored. The white-light PSF was generated by a digital-image-processing system from experimental monochromatic wave aberrations corresponding to three different wavelengths. ${ }^{4}$ In addition, by analyzing the defocusing coefficients present in each monochromatic wave aberration, it was possible to correlate differences in the relative positions of reflecting layers in the retina for different wavelengths of incident light. ${ }^{4}$ Finally, two second-order corrections to the OTF results obtained by the double-pass method were considered. The retinal reflection directionality and the Stiles-Crawford effect were introduced into the OTF calculations by means of the wave aberration and apodized pupils. ${ }^{5}$
Functions commonly used in image-quality determinations, such as the PSF and the OTF, can be computed from the wave aberration when the optical system of the eye is subject to different conditions. In this way, the eye's image quality can be predicted in a large variety of situations. This is useful in order to avoid experimental difficulties and to shorten the duration of the experiments when the image quality of individual eyes must be assessed or controlled under different optical conditions.

As an example of the potential applications of the wave aberration in physiological optics, the PSF is now computed for different levels of luminance, that is to say, with different pupil sizes, and with different states of focus, as might be obtained with different ophthalmic corrections. From these foveal PSF data, retinal images of extended tests in the central area of the fovea, where the isoplanatism condition is maintained, are also calculated. The interest in the retinal images of extended tests is increasing owing to their use in more sophisticated psychophysical experiments.

Preprocessed images of extended test objects are computed below. These objects are filtered by Wiener filters ${ }^{6}$ adapted to the specific OTF of any individual eye. It is shown that the retinal images of these test objects contain more high-spatial-frequency information than the retinal images of the original test objects. By this method, it is possible partially to bypass the degradation caused by the dioptrics of the eye. This ability could be used in subsequent psychophysical experiments in which the effect of the eye's optical system must be eliminated or at least reduced. In what follows, the computational procedure, computergenerated retinal images, and future prospects of the research are presented.

\section{COMPUTATION OF FOVEAL RETINAL IMAGES UNDER DIFFERENT IMAGE-QUALITY CONDITIONS}

In this section, the influence of two important parameters in the optical image quality of the human eye is shown: the mean level of luminance, which is related to the pupil size of the subject, and the state of focus, which is related to oph- 
thalmic corrections. All the calculations have been carried out by using experimental data corresponding to several emmetropized subjects. The results presented here correspond to a limited number of subjects, but the methodology can be systematically applied to a larger range of subjects and conditions.

The pupil size plays an important role in the optical image quality of the eye. Campbell and Gubisch ${ }^{7}$ measured the line-spread function by a double-pass method with artificial pupils of different sizes. They compared these one-dimensional results with those expected for a diffraction-limited eye and showed that, for pupil diameters smaller than $2 \mathrm{~mm}$, the optical system of the eye was limited by diffraction alone. However, there are many practical situations in which larger pupils are present. A two-dimensional analysis of this problem, including a special consideration of the
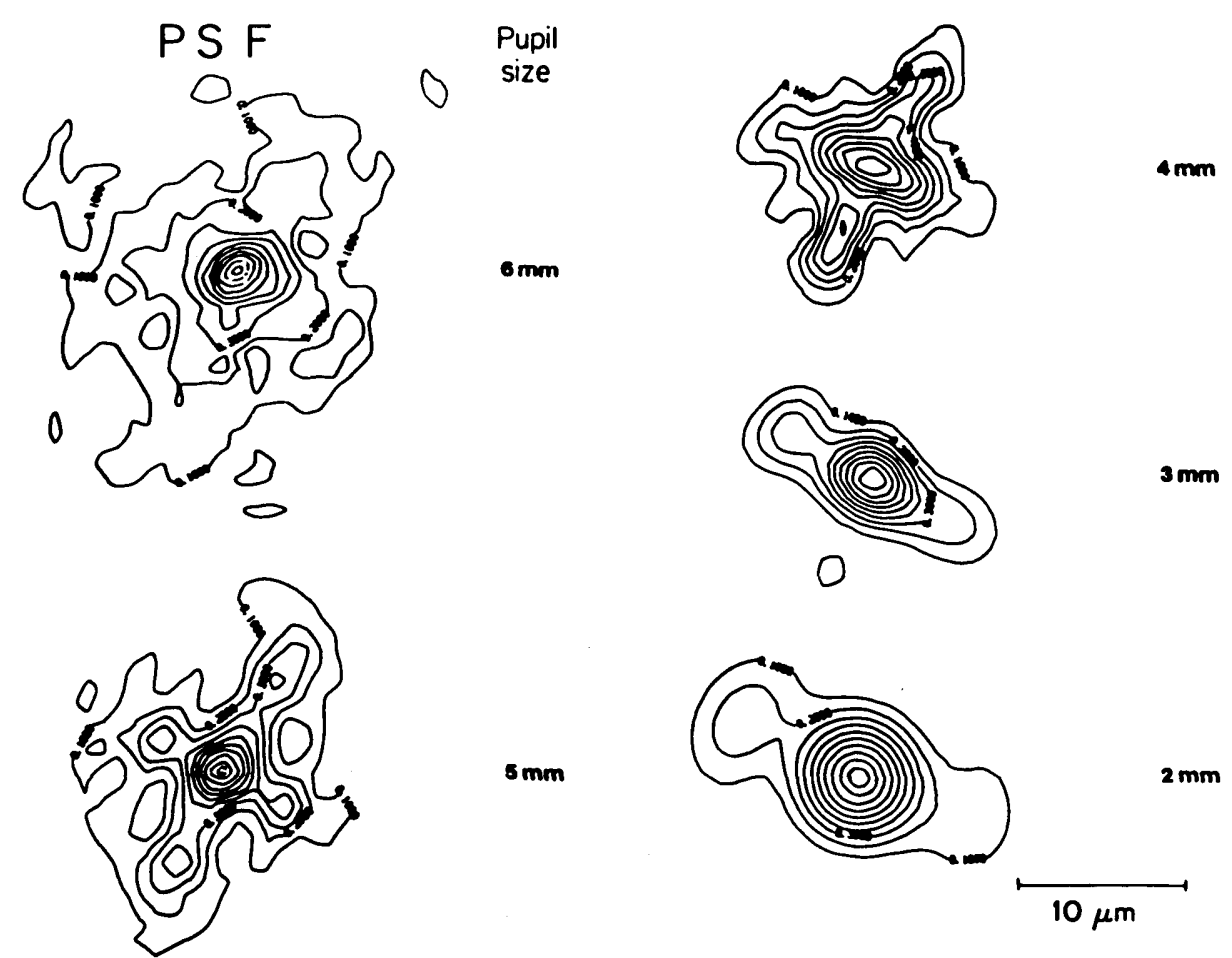

Fig. 1. Contour plot at $I_{\max } / 10$ intervals of the two-dimensional PSF of the eye of a normal and emmetropic subject (PA) with a slight residual astigmatism. It has been computed from the wave-aberration function with values of the pupil diameters from 2 to $6 \mathrm{~mm}$.

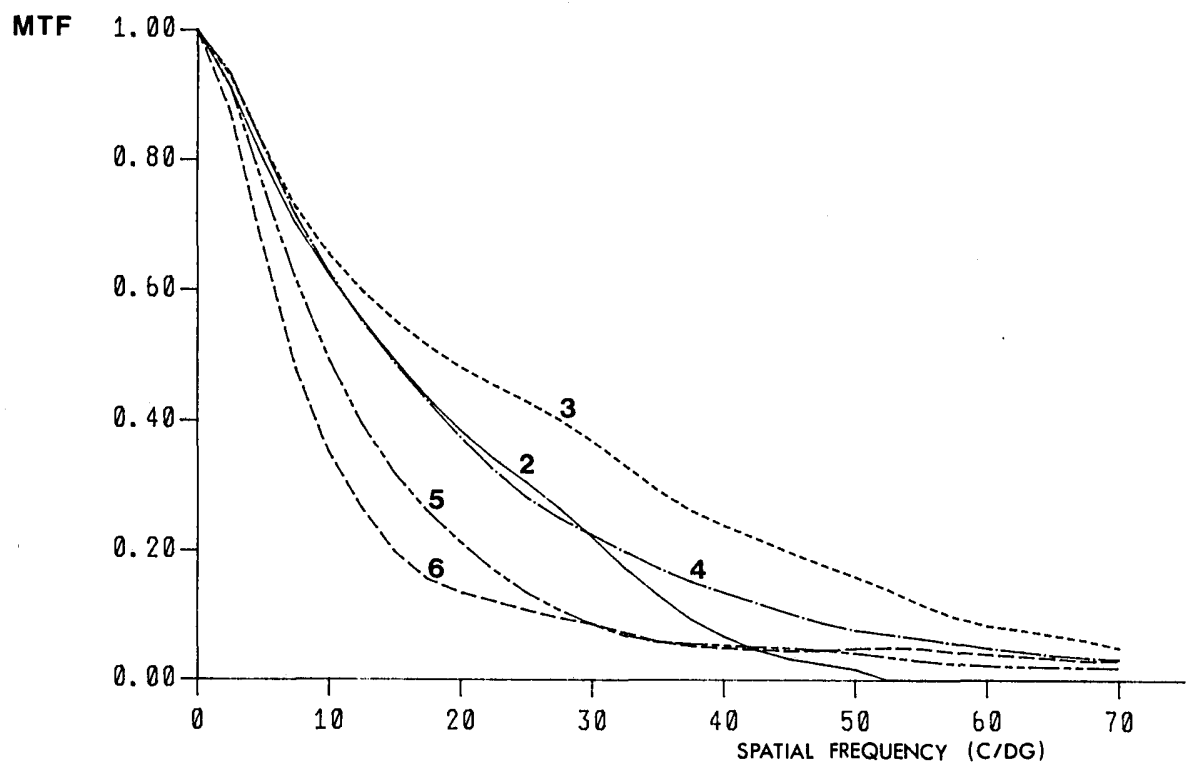

Fig. 2. Sections of two-dimensional modulation transfer functions (MTF's) at 0 deg for pupil diameters (millimeters). (Numbers on curves indicate diameter in millimeters; $\mathrm{C} / \mathrm{DG}$ is cycles per degree.) 
asymmetries, is interesting. The influence of the pupil size is considered by computing the PSF $\left[P_{s}\left(x^{\prime}, y^{\prime}\right)\right]$ from the wave-aberration function $[W(x / R, y / R)]$ by using the expression

$$
\begin{aligned}
P_{s}\left(x^{\prime}, y^{\prime}\right)=\mid \frac{1}{R^{2}} \int & \int_{x^{2}+y^{2} \leq R^{2}} \exp \left[\left(i \frac{2 \pi}{\lambda}\right) W(x / R, y / R)\right] \\
& \times\left.\exp \left[-i \frac{2 \pi}{\lambda f^{\prime} R}\left(x x^{\prime}+y y^{\prime}\right)\right] \mathrm{d} x \mathrm{~d} y\right|^{2},
\end{aligned}
$$

where $x$ and $y$ are pupil plane coordinates, $x^{\prime}$ and $y^{\prime}$ retinal image plane coordinates, $\lambda$ is the wavelength, $R$ is the radius of the pupil, and $f^{\prime}$ is the mean focal length of the human eye. The two-dimensional PSF's, computed from the wave aberration for values of the pupil size varying from 2 to $6 \mathrm{~mm}$, for an emmetropic subject (PA), are shown in Fig. 1. The characteristic evolution of the asymmetric aberrations present in these images should be noted: the PSF's become increasingly asymmetric as the pupil diameter increased from 2 to 6 $\mathrm{mm}$. The OTF has been obtained also from the PSF for these pupil diameters. Sections of the modulation transfer function at 0 deg are plotted in Fig. 2. To study the image quality as a function of pupil size, a commonly used parameter, the Strehl ratio, ${ }^{8}$ has been calculated from the PSF results for different subjects and pupil sizes. The Strehl ratio is taken here as the maximum value of the PSF when the energy in the retinal plane is maintained constant; this result is not divided by the diffraction-limited peak value. Figure 3 shows the inverse of the Strehl ratio as a function of the pupil size for two emmetropic subjects and the aberration-free system. The maximum image quality in terms of the Strehl ratio occurs for approximately 3-mm pupil diameters in most of the subjects considered. At this pupil size, there is an optimal balance between the effects of the aberration and diffraction. Another parameter that contains global information about the asymmetric aberrations has been computed from the PSF for different pupil sizes. This parameter is defined as the mean-squared difference between the actual PSF and a symmetric PSF with the same average spread of light. Results for two subjects are presented in Fig. 4. The value of this parameter increases monotonically for all subjects as the pupil diameter increases from $3 \mathrm{~mm}$. Thus the usual one-dimensional image-quality data fail to indicate the lack of symmetry that occurs for pupil sizes larger than $3 \mathrm{~mm}$ in diameter. Many of these results are just a confirmation of what has long been known, 9,10 but these results were obtained by means of a completely objective and direct methodology.

By using the PSF results, retinal image of extended test objects that subtend small angles on the central fovea can be computed. The optical system of the eye working with incoherent light is generally accepted as a linear system in intensity. For an isoplanatic region (the small area of the central fovea), a test object $f\left(x_{0}, y_{0}\right)$ and its foveal retinal image $g\left(x^{\prime}, y^{\prime}\right)$ are related by the expression

$$
g\left(x^{\prime}, y^{\prime}\right)=\iint f\left(x_{0}, y_{0}\right) P_{s}\left(x^{\prime}-x_{0}, y^{\prime}-y_{0}\right) \mathrm{d} x_{0} \mathrm{~d} y_{0},
$$

where $P_{s}\left(x^{\prime}-x_{0}, y^{\prime}-y_{0}\right)$ is the PSF of the eye. The computation of foveal retinal images of extended test objects is especially interesting when such objects are used in psy- chophysical experiments. In this paper, a part of the wellknown painting Guernica by Picasso is used as a test object. This painting was chosen as a test object because of its simplicity and the presence of well-defined edges. The original object is shown in Fig. 5(a). When this object, $10 \mathrm{~cm} \times$ $10 \mathrm{~cm}$ in size, is viewed by an emmetropic subject from a 5-m distance, its geometrical image is $345 \mu \mathrm{m} \times 345 \mu \mathrm{m}$ (1.2 deg $\times$ $1.2 \mathrm{deg}$ ) on the fovea. The retinal images of this object formed in monochromatic light $(632.8 \mathrm{~nm})$ for an emmetropic subject (PA) with 3- and 6-mm pupil diameters are presented in Figs. 5(b) and 5(c), respectively. It has been assumed that the energy is maintained constant during the incoherent image-formation process that is practically correct in normal subjects.

Another optical factor influencing the image quality that is considered in this section is the state of focus that is directly related to any ophthalmic correction in use.

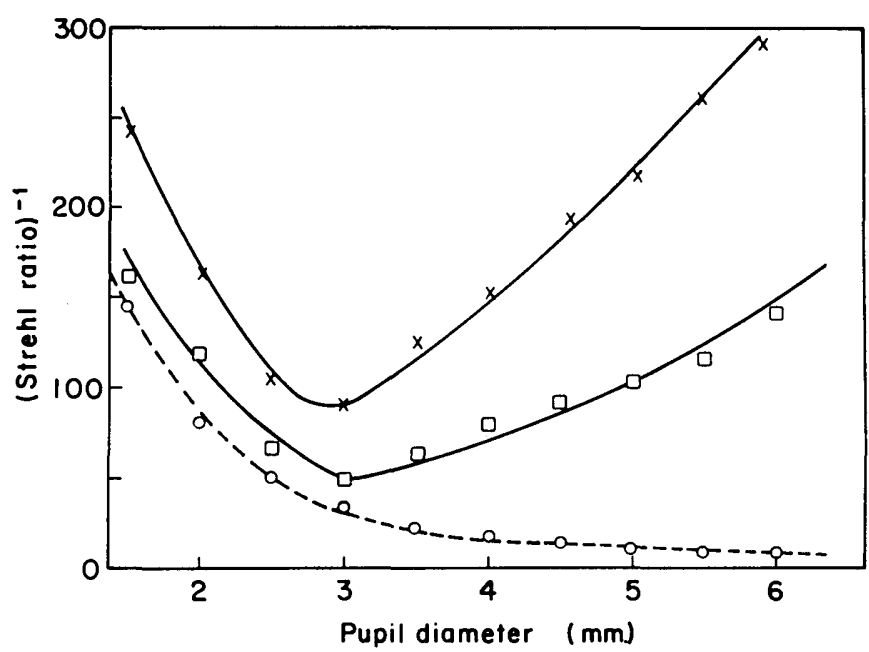

Fig. 3. The inverse of the Strehl ratio as a function of the pupil size for two emmetropic subjects, PA (- - ) and JS $(\rightarrow-)$, and a diffraction-limited system (-- - ).

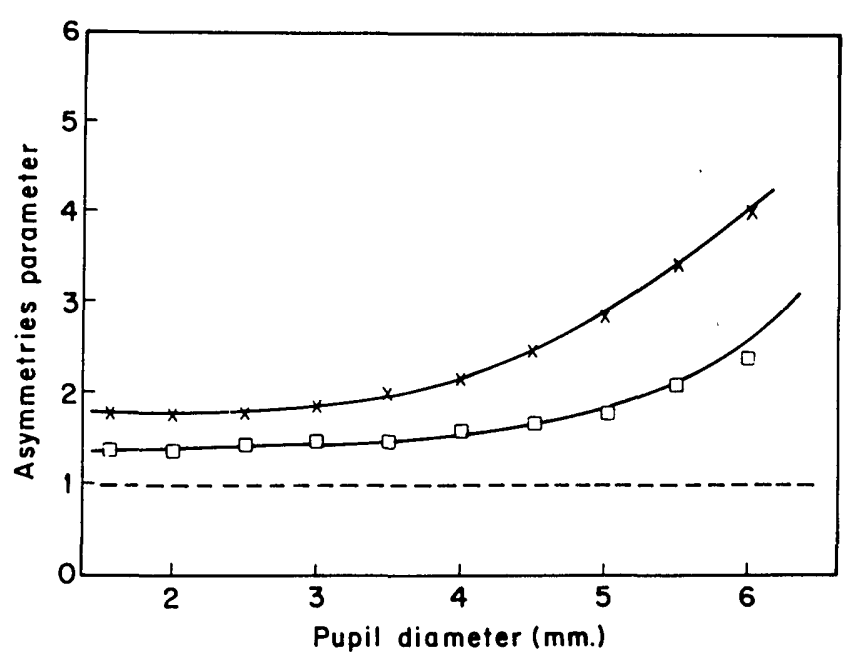

Fig. 4. Variations of an asymmetry parameter computed for two individual eyes and for an aberration-free system (-..-). 


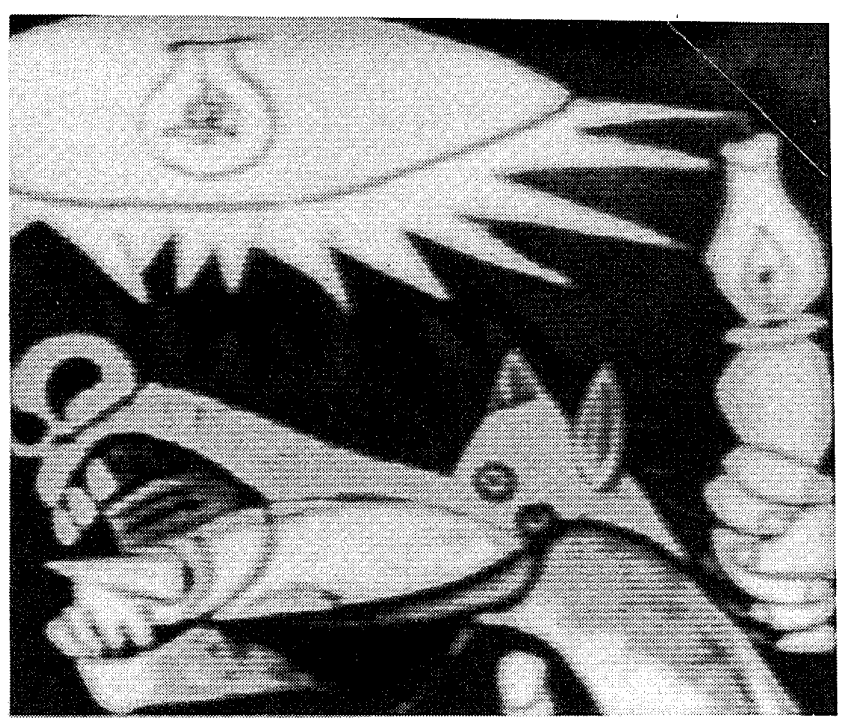

(a)

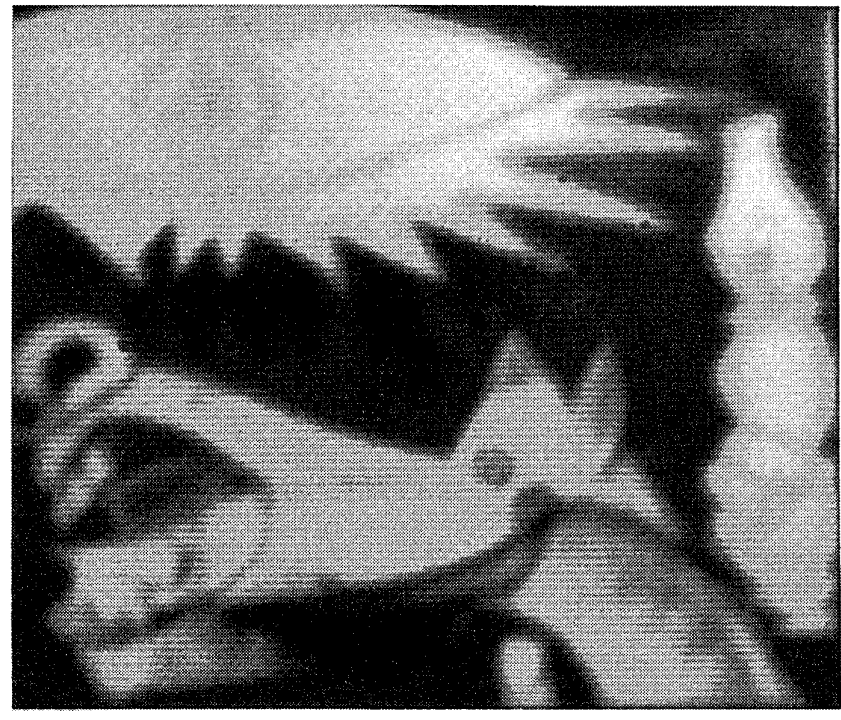

(b)

Changes in focus generate specific two-dimensional characteristics in the PSF, depending on the wave aberration of each subject. The PSF is computed by means of Eq. (1) by using a new wave-aberration function $\left[W_{a}(x / R, y / R)\right]$, obtained when a defocusing factor is added to the retrieved wave aberration $W(x / R, y / R)$ :

$$
W_{a}(x / R, y / R)=W(x / R, y / R)+W_{20}\left(x^{2}+y^{2}\right) / R^{2}
$$

The coefficient $W_{20}$ characterizes the amount of defocus. ${ }^{11}$ Figure 6 shows the contour plot of the two-dimensional PSF's computed under different states of focus for a subject with a $3-\mathrm{mm}$ pupil diameter. It can be seen from these results that the image quality of the eye as a function of focus is not directly predictable. The two-dimensional analysis of Fig. 6 shows important asymmetries and the presence of several relative maxima in the PSF's when the amount of

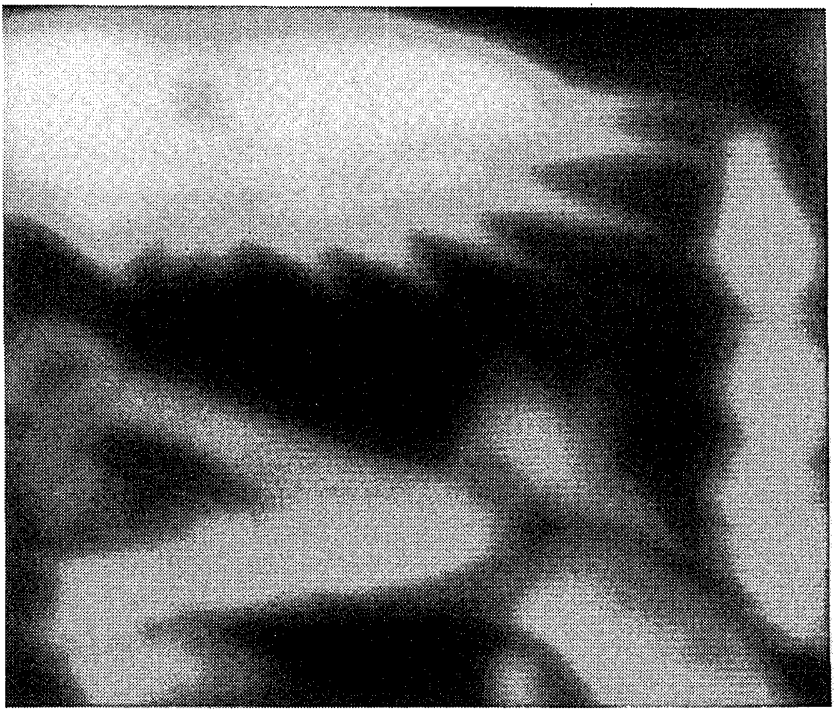

(c)

Fig. 5. (a) Original test object used in the computations. It corresponds to a section of the painting Guernica by Picasso (Prado Museum, Madrid). The geometrical image is $345 \mu \mathrm{m} \times 345 \mu \mathrm{m}(1.2$ $\operatorname{deg} \times 1.2 \mathrm{deg}$ ) on the central fovea. (b) Foveal retinal image of the original object presented in (a) when it is illuminated in red light $(632 \mathrm{~nm})$ and viewed by an emmetropic subject with a 3-mm-diameter pupil. (c) Foveal retinal image of the original object in (a) viewed by the same subject as in (b) but with a 6-mm-diameter pupil.

defocus is increased. In fact, the presence of a slight residual astigmatism in the subject's eye should be noted.

By using Eq. (1), one can compute the PSF with the pupil size $(R)$ and the state of focus $\left(W_{20}\right)$ as variables. From these results, the Strehl ratio has been obtained as a function of $R$ and $W_{20}$. These results for two different subjects are plotted in Fig. 7. The best image quality depending on the state of focus is not the same for each pupil size. From 2- to 6-mm pupil diameter, the difference in the position of the maximum Strehl ratio is approximately $1 \mathrm{D}$. This could be related to the induced myopia at low luminances. ${ }^{12,13}$ The influence of aberrations in night myopia has traditionally been proposed to explain this phenomenon. The depth of focus for each pupil diameter has also been obtained from the results of Fig. 7. The depth of focus has been defined at a given pupil size as the dioptric range in which the relative Strehl ratio is greater than 0.2 . The mean results obtained 


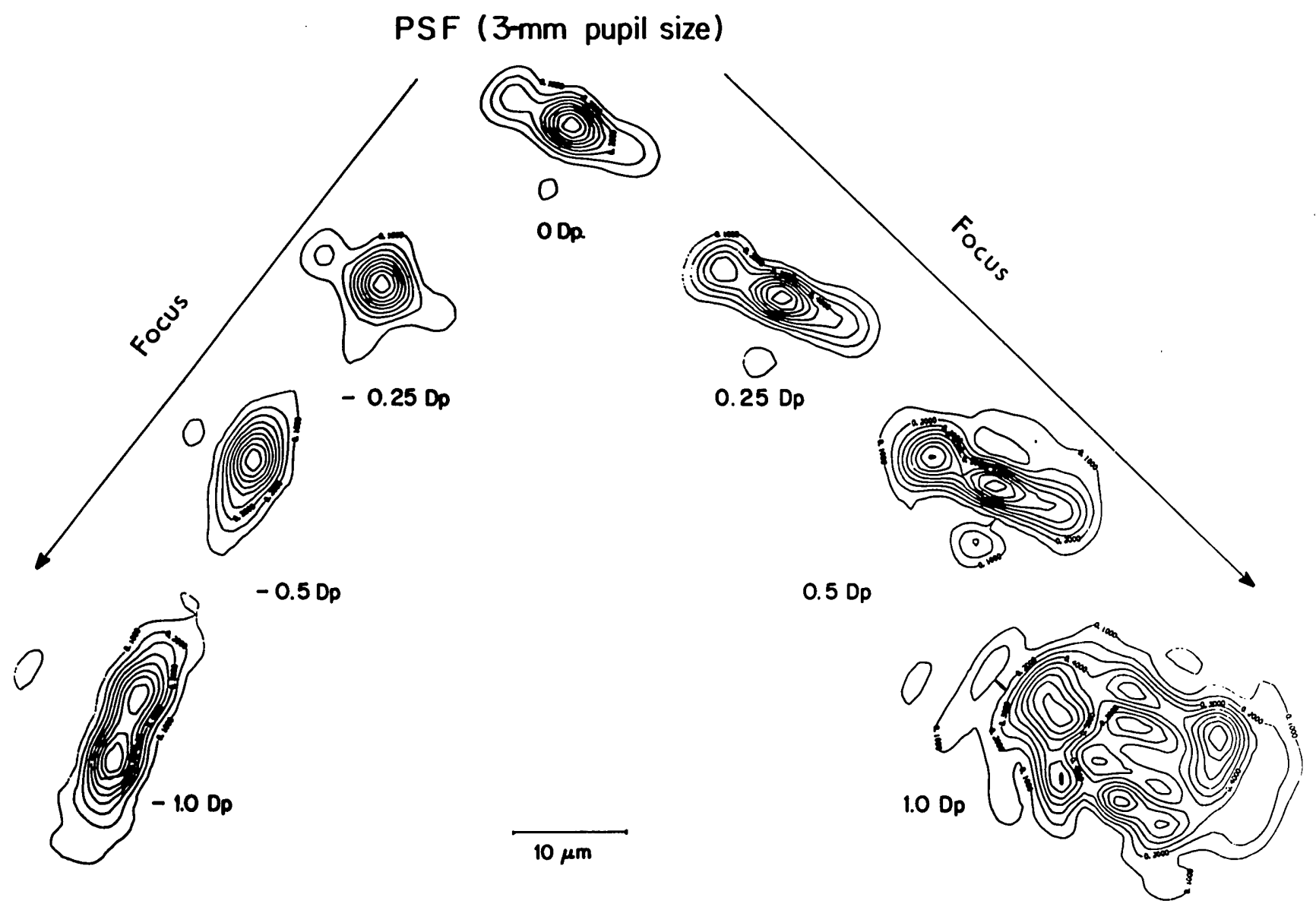

Fig. 6. Contour plots of the bidimensional PSF of the eye of an emmetropic subject (PA) with a slight residual astigmatism with 3-mm pupil diameter under different states of focus from -1 to $1 \mathrm{D}$.

in five emmetropic subjects are shown in Fig. 8. Knowledge of the effect of defocus on the retinal image is important in relation to its global influence on contrast perception. ${ }^{14}$

By means of Eq. (2), the retinal images of the original object of Fig. 5(a) have been computed under two different states of focus. Figure 9(a) is the foveal retinal image for subject PA with 3-mm pupil diameter and 0.25-D shift in focus, and Fig. 9(b) is for the same subject with 1-D change in focus. Owing to the several relative maxima in the defocused PSF (Fig. 6), the retinal image of Fig. 9(b) presents a double image, distinguishable mainly in the edges of the figure.

\section{RETINAL IMAGES OF PREFILTERED TEST OBJECTS}

In the previous section the degradation that is introduced by the dioptrics of the eye under different conditions was characterized by means of the PSF. Here, a digital prefiltering of test objects by using Wiener filters ${ }^{6}$ adapted to the OTF of any individual eye is proposed to overcome this degradation. Wiener filtering is a well-known image-processing technique. ${ }^{6}$ It is the optimum filtering that minimizes the mean-squared difference between the original and the restored images. Its functional dependence is given by

$$
F_{w}(u, v)=\frac{H^{*}(u, v)}{|H(u, v)|^{2}+\frac{\phi_{n}(u, v)}{\phi_{f}(u, v)}},
$$

where $H(u, v)$ is the OTF of the eye and $\phi_{n}(u, v)$ and $\phi_{f}(u, v)$ are the power spectra of noise and the original image. If we assume that $\phi_{n}(u, v)$ and $\phi_{f}(u, v)$ are white, $\phi_{n}(u, v) / \phi_{f}(u, v)$ in Eq. (4) can be taken as a constant adjustable parameter. This is a reasonable approximation when the aberrations of the system are severe compared with the variations of $\phi_{n}(u$, $v)$ and $\phi_{f}(u, v)$. The procedure presented here is based on the computation of new test objects $\left[f_{w}\left(x_{0}, y_{0}\right)\right]$ preprocessed by using a Wiener filter with an appropriate constant parameter by the expression

$$
f_{w}\left(x_{0}, y_{0}\right)=f\left(x_{0}, y_{0}\right) \otimes \mathrm{FT}^{-1}\left[F_{w}(u, v)\right],
$$

where $\otimes$ means convolution and $\mathrm{FT}^{-1}$ is the inverse Fourier transformation. The strength of the filtering depends on the choice of the filter's parameter, which must be related to the spatial characteristics of object test and the OTF of the considered eye. Visual inspection of the resulting filtered images gave the optimal value of the filter's parameter as 0.001 . The foveal retinal image of the new test object $\left[g_{w}\left(x^{\prime}\right.\right.$, 

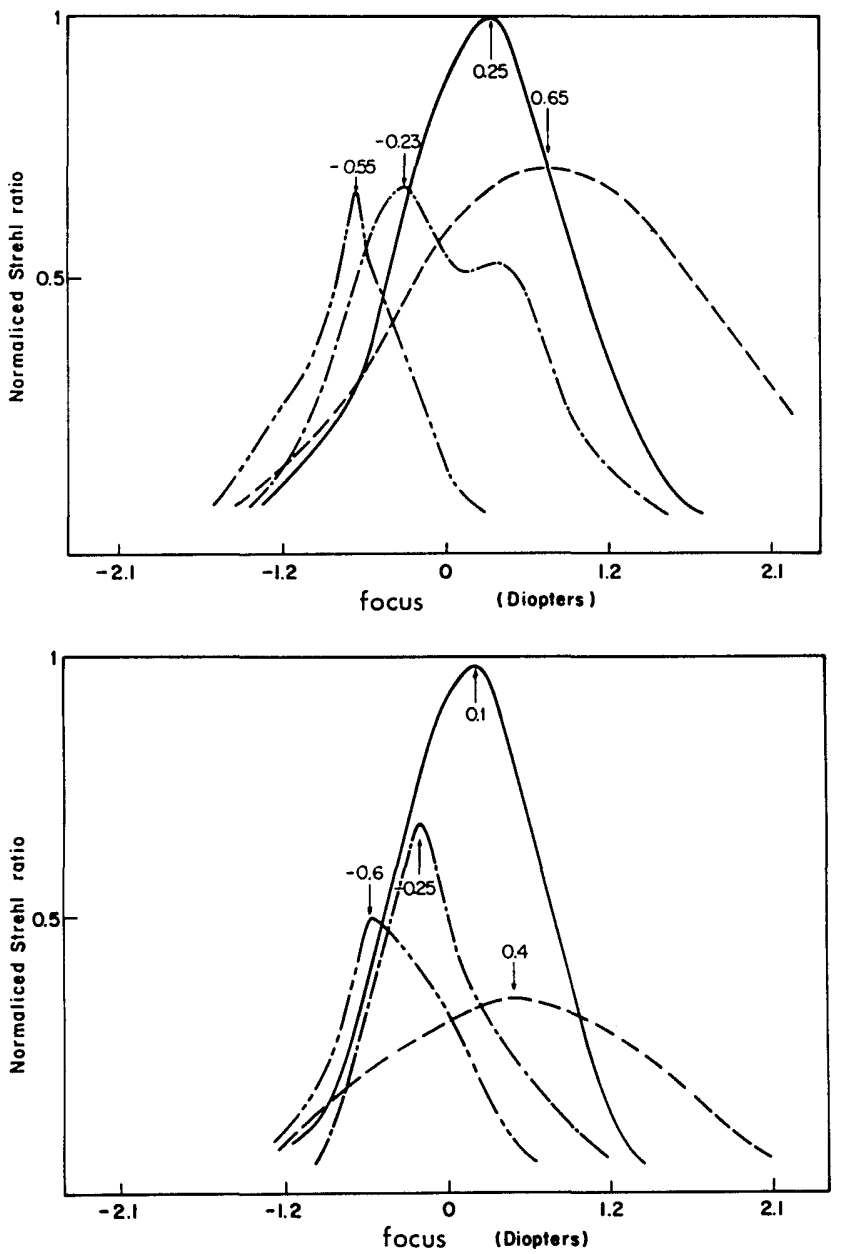

Fig. 7. Comparison of the Strehl ratio for two different subjects with different states of focus and for the following pupil sizes: 2$\mathrm{mm}$ pupil diameter $(--), 3-\mathrm{mm}$ pupil diameter $(-), 4-\mathrm{mm}$ pupil diameter (- - ), and 5-mm pupil diameter (- - -). The figures on curves indicate the optimal focus (diopters) for each pupil diameter.

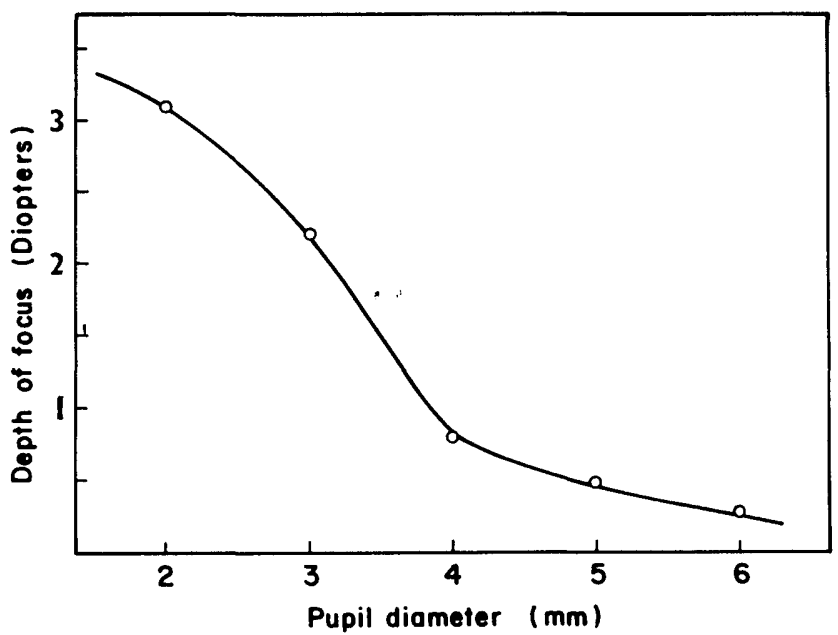

Fig. 8. Depth of focus as a function of pupil size. The mean results for five emmetropic subjects computed from their wave-aberration results. $\left.\left.y^{\prime}\right)\right]$ is computed by Eq. (2). The prefiltered object $\left[f_{w}\left(x_{0}\right.\right.$, $\left.y_{0}\right)$ ] is shown in Fig. 10(a) [compare it with Fig. 5(a)], and its retinal image $\left[g_{w}\left(x^{\prime}, y^{\prime}\right)\right]$ for a $6-\mathrm{mm}$ pupil diameter is in Fig. 10(b). The retinal image of the new test object contains more high-spatial-frequency information than the retinal image of the original test object. To note the improvement, we must compare the retinal images of both the original test object [Fig. 5(c)] and the prefiltered test object [Fig. 10(b)]. A partial bypass of the spatial degradation introduced by the optics of the eye is obtained. This could be useful in psychophysical experiments in which complex test objects are involved and in which, in most cases, the effect of the eye's

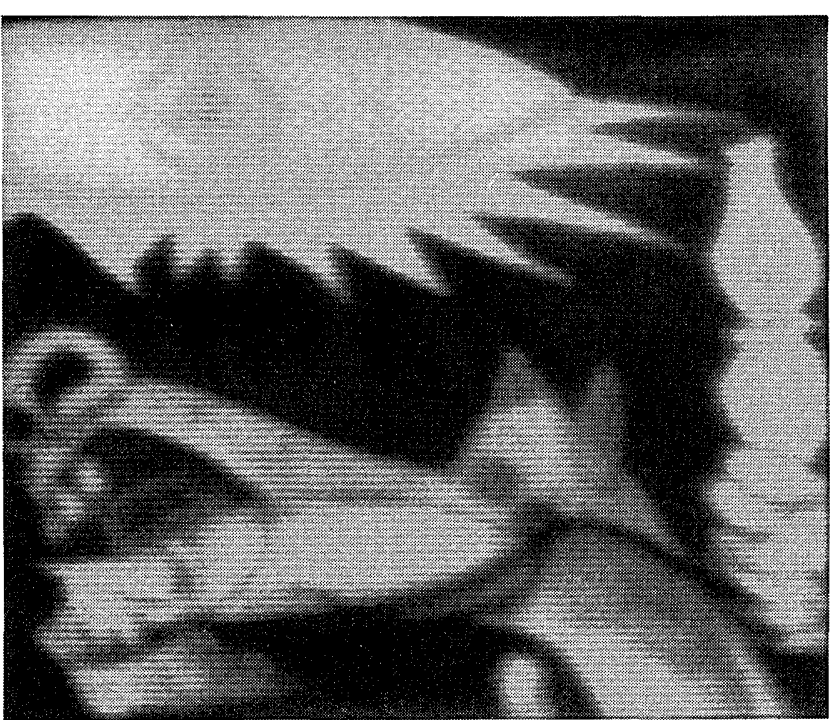

(a)

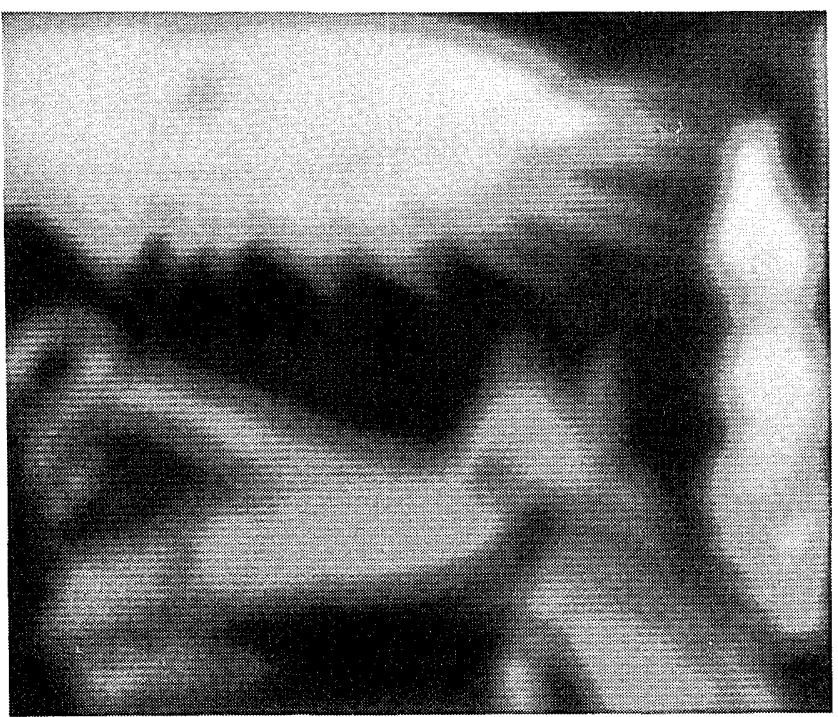

(b)

Fig. 9. (a) Retinal image of the original test object of Fig. 5(a) for an emmetropic subject (PA) with $-0.25 \mathrm{D}$ of defocus. (b) Retinal image of the original object of Fig. 5(a) for an emmetropic subject (PA) with $-1 \mathrm{D}$ of defocus. 


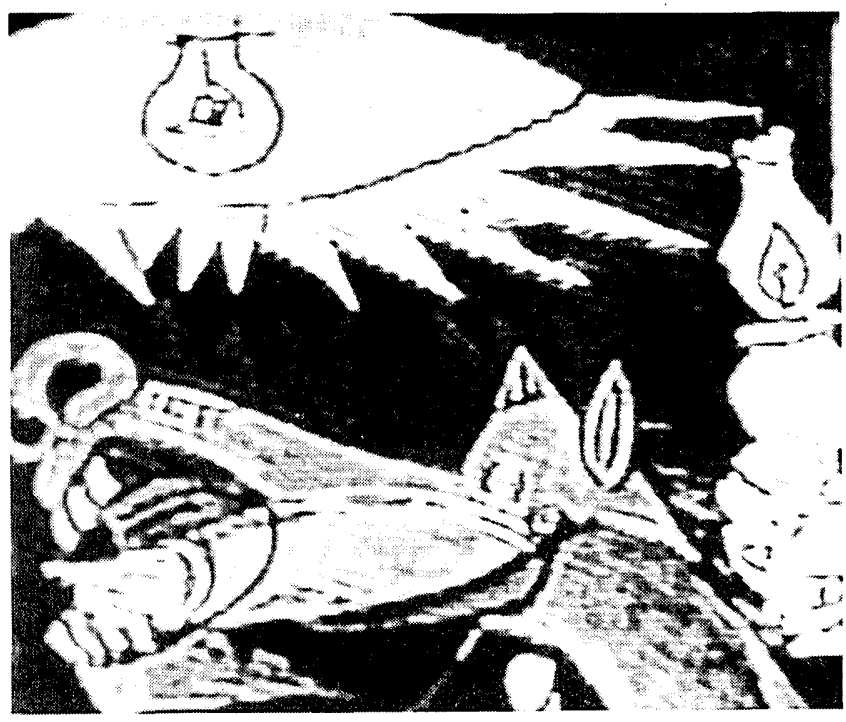

(a)

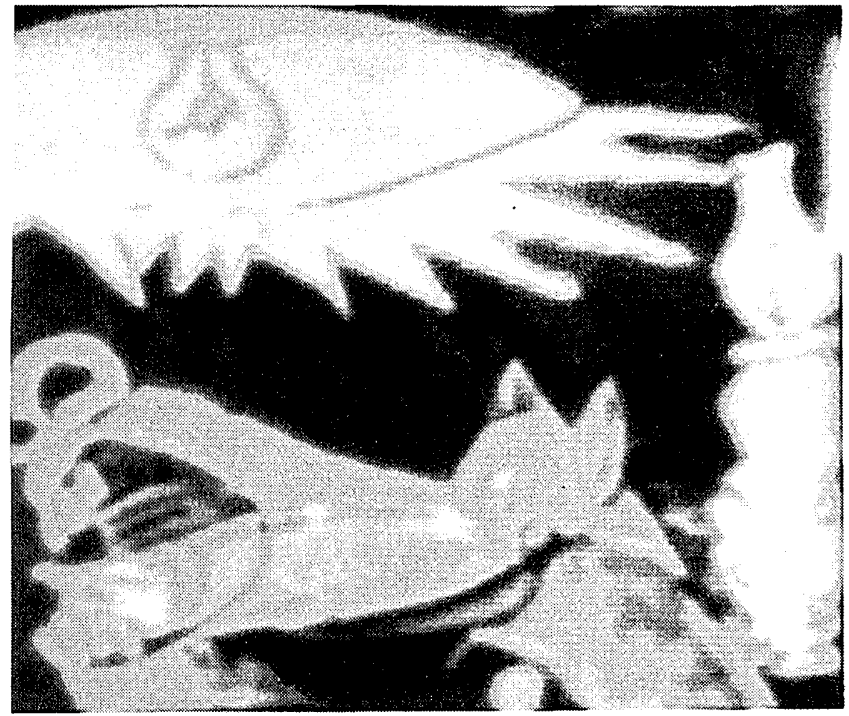

(b)

Fig. 10. (a) Prefiltered image computed from the original Fig. 5(a) by means of a Wiener filter adapted to the OTF of the subject PA for a 6-mm pupil with a value of the filter's parameter of 0.001 . (b)

Foveal retinal image of the prefiltered test (a) obtained under the same conditions of image quality (6-mm pupil diameter) as that presented in Fig. 5(c).

optics is undesirable and generally is not precisely controlled.

Image-enhancement techniques have already been proposed to compensate for the degradation introduced by the whole visual system. Peli and $\mathrm{Peli}^{15}$ used measurements of the contrast sensitivity function as a modulation transfer function to improve the visual response in low-vision patients by image-processing techniques. The linear method proposed here provides just a partial compensation of the optical degradation introduced by a human eye. A detailed psychophysical study of this method could evaluate its potential application in vision research. Here, I have merely outlined the procedure and preliminary results to show the possibilities of the technique.

\section{SUMMARY AND PROSPECTS}

In this paper, computer-generated retinal images for foveal targets have been presented. The influence of important factors on the image quality of the eye has been introduced. From the PSF obtained by using a hybrid optical-digital method, the wave aberration of the eye was retrieved. By using this function, the effect of pupil size and state of focus were introduced into the computations. This methodology permits the PSF to be obtained under different conditions without uncomfortable repetition of experimental measurements. In addition, the duration of the experimental procedure to assess the image quality and its evolution with several parameters can be drastically reduced. Assuming isoplanatism in an area of the central fovea, the retinal images of extended tests objects were computed by direct convolution by the PSF. New test objects have been computed by Wiener filtering adapted to the specific OTF of any individual eye. It is shown that the retinal images of these tests contain more high-spatial-frequency information than the retinal images corresponding to the original object. By this method, a partial compensation of the degradation caused by the eye's optics is obtained.

After the optics of the eye, the next step in the visual processing is the sampling in the photoreceptor mosaic. A detailed study of the spatial properties of the sampling requires experimental data of both the cone distribution and the image quality in the living human fovea. However, regarding cone topography, only Williams ${ }^{16}$ has proposed a psychophysical method that permits one to estimate the cone spacing and packing geometry in the living human retina. Recently, with the aim of obtaining direct physical measurements on the spatial distribution of cones, Artal and Navarro ${ }^{17}$ have proposed a high-resolution method, based on speckle interferometry, to measure the cone spacing in the living fovea. The result in the central foveola for a normal subject was above $0.51 \mathrm{arcmin}(2.45 \mu \mathrm{m}$ in the retina). Further improvements of this method could permit us a better understanding of the retinal photoreceptors' organization.

By using these new structural data in conjunction with those of image quality, a precise evaluation of the spatial sampling could be carried out. Such an evaluation is important in relation to the study of the physical limits of visual resolution, aliasing in the retinal sampling, and the generation of postsampling retinal images of real tests.

\section{ACKNOWLEDGMENTS}

I thank J. Santamaría and R. Navarro for helpful discussions and A. Plaza and M. Perez for their assistance while this research was in progress. This research was supported partially by the Comisión Interministerial de Ciencia y Tecnología, Spain. Final versions of the manuscript were written at the Institut d'Optique, Orsay, France; I thank P. Chavel for his hospitality and S. Mallick for his revisions of the English.

\section{REFERENCES}

1. J. Santamaría, P. Artal, and J. Bescós, "Determination of the point-spread function of human eyes using a hybrid opticaldigital method," J. Opt. Soc. Am. A 4, 1109-1114 (1987). 
2. P. Artal, J. Santamaría, and J. Bescós, "Retrieval of the wave aberration of the human eyes from actual point-spread function data," J. Opt. Soc. Am. A 5, 1201-1206 (1988).

3. P. Artal, J. Santamaría, and J. Bescós, "Phase transfer function of human eyes and its influence on the point-spread function and wave aberration," J. Opt. Soc. Am. A 5, 1791-1795 (1988).

4. P. Artal, J. Santamaría, and J. Bescós, "Optical-digital procedure for the determination of the retinal image of a point test in white light," Opt. Eng. 28, 687-690 (1989).

5. P. Artal, "Incorporation of directional effects of the retina into the computations of the optical transfer function of human eyes," J. Opt. Soc. Am. A 6, 1941-1944 (1989).

6. W. K. Pratt, Digital Image Processing (Wiley-Interscience, New York, 1978).

7. F. W. Campbell and R. W. Gubisch, "Optical image quality of the human eye," J. Physiol. (London) 186, 558-578 (1966).

8. M. Born and E. Wolf, Principles of Optics (Pergamon, Oxford, 1970).

9. G. Westheimer, "Pupil size and visual resolution," Vision Res. 4, 39-45 (1964).
10. F. W. Campbell and D. G. Green, "Optical and retinal factors affecting visual resolution," J. Physiol. (London) 181, 576-593 (1965).

11. H. H. Hopkins, "The frequency response of a defocused optical system," Proc. R. Soc. London Ser. A 231, 91-102 (1955).

12. J. M. Otero and A. Duran, "Rendimiento fotometrico de sistemas opticos a bajas luminosidades," Anal. Fis. Quim. 37, 459477 (1941).

13. M. Koomen, R. Skolnik, and R. Tousey, "A study of night myopia," J. Opt. Soc. Am. 41, 80-90 (1951).

14. G. Legge, K. T. Mullen, G. C. Woo, and F. W. Campbell, "Tolerance to visual defocus," J. Opt. Soc. Am. A 4, 851-863 (1987).

15. E. Peli and T. Peli, "Image enhancement for visually impaired," Opt. Eng. 23, 47-51 (1984).

16. D. R. Williams, "Topography of the foveal cone mosaic in the living human eye," Vision Res. 28, 433-454 (1988).

17. P. Artal and R. Navarro, "High-resolution imaging of the living human fovea: measurement of the intercenter cone distance by speckle interferometry," Opt. Lett. 14, 1098-1100 (1989). 


.




\section{Grating Diffraction}

FEATURE EDITORS

Thomas K. Gaylord Elias N. Gyytsis

School of Electrical Engineering Georgia Institute of Technology

Atlanta, Georgia 30332-0250 